Umwelt und Entwicklung, Rio : Fünf Jahre danach

\title{
Umwelt, Entwicklung und Zusammenarbeit : eine Standortbestimmung
}

Franco Romerio and Milad Zarin-Nejadan

\section{(2) OpenEdition}

1 Journals

Electronic version

URL: http://journals.openedition.org/sjep/746

DOI: 10.4000/sjep.746

ISSN: 1663-9677

Publisher

Institut de hautes études internationales et du développement

Printed version

Date of publication: 1 mars 1997

Number of pages: $189-210$

ISSN: 1660-5926

\section{Electronic reference}

Franco Romerio und Milad Zarin-Nejadan, « Umwelt, Entwicklung und Zusammenarbeit : eine Standortbestimmung », Schweizerisches Jahrbuch für Entwicklungspolitik [Online], 16 | 1997, Online erschienen am: 13 August 2012, abgerufen am 07 September 2020. URL : http://

journals.openedition.org/sjep/746; DOI : https://doi.org/10.4000/sjep.746 


\section{UMWELT, ENTWICKLUNG UND ZUSAMMENARBEIT : EINE STANDORTBESTIMMUNG}

\section{EINLEITUNG}

Bis zu Beginn der siebziger Jahre galt die Sorge um die Umweltqualität als Luxus, den sich die Entwicklungsländer schlicht und einfach nicht leisten konnten. Anlässlich der Konferenz der Vereinten Nationen in Stockholm im Jahre 1972 machte sich die indische Premierministerin Indira Gandhi zur Sprecherin zahlreicher Entwicklungsländer, indem sie erklärte : "Wie kann man von den Bewohnern der Dörfer und Slums verlangen, sie sollten etwas gegen die Verschmutzung der Luft, der Flüsse und der Meere tun, wenn sie ums eigene Überleben kämpfen? Sind nicht das Elend der Armen und die Habgier der Reichen die schlimmsten Übel ? » Zumindest bis zu einem bestimmten Grad wurde damals der Umweltschutz als Hindernis für die wirtschaftliche Entwicklung betrachtet.

Zwanzig Jahre später, im Juni 1992, versammelten sich über 30'000 Personen in Rio de Janeiro, um der Umwelt- und Entwicklungskonferenz der Vereinten Nationen (UNCED), « Erdgipfel » genannt, beizuwohnen. Vertreter von mehr als 180 Nationen nahmen an der Konferenz teil, darunter ungefähr 120 Staats- und Regierungschefs, Nichtregierungsorganisationen (NROs), Vertreter der Industrie, religiöse und ethnische Gruppen, Erzieher und Studierende. Zweifellos handelte es sich bei diesem Gipfel um ein Ereignis, das aufgrund seiner Grösse, seiner Tragweite und der erzeugten Erwartungen alles Bisherige in den Schatten stellte. Vergleicht man die damaligen und heutigen Thesen über die Vereinbarkeit von wirtschaftlicher Entwicklung und Umweltschutz, so wird deutlich, welcher Weg in den vergangenen 25 Jahren zurückgelegt wurde. Ein vorsichtiger Umgang mit der Umwelt und den natürlichen Rohstoffen wird nicht mehr als Hindernis für die Entwicklung betrachtet, sondern als eine Voraussetzung dafür, und nimmt in sämtlichen Programmen zur Verbesserung der Lebensqualität der Armen dieser Welt einen wichtigen Platz ein.

Ziel dieses Beitrags ist es, die vielfältigen Interaktionen zwischen Umwelt und wirtschaftlicher Entwicklung aufzuzeigen und einen Überblick zu vermitteln über die konkreten Möglichkeiten, ökologische Gesichtspunkte in den Ländern des Südens bei der Zusammenarbeit und der Entwicklungshilfe zu berücksichtigen. Der Beitrag ist in vier Teile gegliedert. Anschliessend an diese kurze Einleitung wird in Teil 2 ein Überblick über die komplexen und oft mehrdeutigen Interdependenzen zwischen Umwelt und Entwicklung geboten. In Teil 3 werden die Mittel diskutiert, die den Industrieländern und der Völkergemeinschaft zur Verfügung stehen, um Programme auf lokaler und globaler Ebene zur Gesundung der Umwelt in den Ländern des Südens durchzuführen oder zu fördern. In

* Der Dank der Autoren geht an Beat Bürgenmeier und an das Redaktionskomitee des Jahrbuchs für ihre Bemerkungen und Anregungen. 
Teil 4 werden zusammenfassend die wichtigsten Ergebnisse dieser Analyse diskutiert.

\section{INTERDEPENDENZEN ZWISCHEN UMWELT UND ENTWICKLUNG}

Im folgenden sollen kurz die zahlreichen Interdependenzen zwischen Entwicklung und Umwelt aufgezeigt werden. ' Die Diskussion erfolgt absichtlich auf allgemeiner Ebene, da die aufgeworfenen Fragen sowohl für Industrieländer als auch für Entwicklungsländer Gültigkeit besitzen.

\subsection{PROBLEMATIK UND LÖSUNGSANSÄTZE}

Wirtschaftliche Entwicklung wurde in der Vergangenheit oft mit Degradierung der Umwelt gleichgesetzt. Dass dabei aber Umweltprobleme von derartiger Tragweite entstehen könnten, dass sie ein regelrechtes Hindernis für die Weiterentwicklung darstellen, wurde nie ernsthaft in Betracht gezogen. Generell wurde dieses Problem als Schwierigkeit im kleinen Massstab ${ }^{2}$ wahrgenommen, zumal sich die Schäden vorrangig auf dieser Ebene bemerkbar machten. Aber auch auf dieser Ebene wurden nur die gravierendsten Probleme wahrgenommen. Gründe dafür waren zum einen das Fehlen wissenschaftlicher Erkenntnisse und zum andern das kulturell bedingte geringe Gewicht, welches Umweltfragen beigemessen wurde. Sobald das Ausmass der Schäden die Öffentlichkeit in Alarmbereitschaft versetzte, wurden zumindest in den Industrieländern technische, legislatorische oder, was seltener der Fall war, wirtschaftliche Massnahmen ergriffen. Dennoch waren diese Probleme in den Augen der Verantwortlichen nie mehr als "Zwischenfälle », die anhand von Einzelmassnahmen behoben werden konnten. Ein Beispiel hierfür wäre die Luftverschmutzung in London, die durch die Verbrennung von Kohle in Industrie und Haushalten und insbesondere durch die Partikelkonzentration in der Luft hervorgerufen wurde und die Anfang Dezember 1952 die Sterblichkeit sprunghaft ansteigen liess. Die britische Regierung sah sich gezwungen, legislatorische Massnahmen zu ergreifen, denen zumindest ein teilweiser Erfolg beschieden war. ${ }^{3}$ Schliesslich wurde der Notstand aufgehoben, aber erst zu Beginn der neunziger Jahre wurde die Diskussion um die durch Partikelkonzentration bedingte Luftverschmutzung erneut aufgegriffen. ${ }^{4}$

Seit einigen Jahren werden die sehr engen und ausserordentlich komplexen Zusammenhänge zwischen Umwelt und Entwicklung nicht mehr in Frage gestellt. Die Öffentlichkeit ist sich des Ausmasses der Umweltdegradierung bewusst geworden, vor allem, weil sie nicht nur im kleinen, sondern auch im mittleren und im grossen Massstab offensichtlich wird, sowie aufgrund ihrer Auswirkungen auf Gesundheit, Lebensqualität, Wirtschaft und Gesellschaft. Es

\footnotetext{
1 vgl. dazu Weltbank, Rapport sur le développement dans le monde 1992 - Le développement et l'environnement, Washington, 1992, sowie die Sondernummer der Revue Tiers Monde zum Thema « Environnement et développement 》 (Band 33, Nr. 130, 1992)

2 Man unterscheidet zwischen « kleinem Massstab » (z.B. die Luftverschmutzung in einer Stadt), " mittlerem Massstab (Waldsterben) und « grossem Massstab» (Treibhauseffekt).

$3 \mathrm{Vgl}$. Ministry of Housing and Local Government, Report of the Committee on Air Pollution (Beaver Report), London, HMSO, 1954

4 Vgl. A. Seaton et al., « Particulate Air Pollution and Acute Health Effects », The Lancet, 345, 21. Januar 1995
} 
hat sich die Erkenntnis durchgesetzt, dass die Ursachen zahlreicher Probleme nicht in Ausrutschern und Zwischenfällen, sondern im sozioökonomischen Entwicklungsprozess selbst liegen. Heute steht fest :

- dass bei der Entwicklung der nördlichen Industrieländer die ökologische Komponente vollumfänglich integriert werden muss,

- dass die Entwicklung neu überdacht werden muss, da es nicht genügt, die Umwelt als neuen Parameter der Problematik beizufügen,

- dass dabei umso mehr Eile geboten ist, als sich die Länder des Südens sowie die ehemaligen kommunistischen Länder das Entwicklungsmodell der nördlichen Industrieländer zum Vorbild genommen haben.

Umwelt und Entwicklung werden heute als zwei voneinander abhängige Komponenten derselben Problematik betrachtet. Ein Entwicklungsprozess, bei dem Umweltprobleme ausser acht gelassen und die in der Vergangenheit getroffenen Entscheidungen nicht neu überdacht werden, wird nicht nur aufgrund verschlechterter Lebensbedingungen und Klimaveränderungen sich in seiner eigenen Entfaltung behindern, sondern zudem mangels Ressourcen und Raum für die verursachten Abfälle an seine Grenzen stossen. Grundsätzlich stellt sich die Frage nach den Folgen für die Umwelt und nach den Grenzen der Entwicklung, vor allem, wenn die Mehrheit der Weltbevölkerung die Konsumgewohnheiten der Industrieländer imitiert, die ihr Wachstum ohne Rücksicht auf die Umwelt weiter vorantreiben ${ }^{5}$.

Die Diskussion um die « nachhaltige Entwicklung », die anlässlich der UNCED 1992 internationales Aufsehen erregte, fügt sich in diesen Kontext ein ${ }^{6}$. Die Überlegungen zur nachhaltigen Entwicklung haben zum Ziel, Modelle zur wirtschaftlichen und sozialen Entwicklung des Planeten zu erarbeiten, die mit der Umwelt im Einklang stehen. Dabei werden Entwicklungsmodelle aus der Vergangenheit mehr oder weniger explizit auf ihre Fähigkeit hin untersucht, die ökologische Dimension in ihre Funktionsweise $\mathrm{zu}$ integrieren. Eine langfristige Sichtweise soll das Schicksal zukünftiger Generationen berücksichtigen. Besondere Aufmerksamkeit jene Massen von Menschen, die in tiefster Armut, unterernährt, obdachlos, ohne ärztliche Hilfe und ohne Bildung leben.

\subsection{WIRTSCHAFTSWACHSTUM UND UMWELT ${ }^{\prime}$}

Das Wirtschaftswachstum kann zu einer Gefahr für die Umwelt werden. Seine Belastung für die Umwelt kann die Grenze des Erträglichen überschreiten. Andererseits kann Wachstum aber auch zusätzliche Ressourcen zur Finanzierung der Umweltpolitik, für Investitionen in saubere Technologien, für die Bereitstellung notwendiger Infrastrukturen usw. schaffen. Darüber hinaus wäre es unrealistisch zu glauben, man könne die wichtigsten Umweltprobleme der Gegenwart in einer Zeit der wirtschaftlichen Stagnation angehen, es sei denn, es fände ein

\footnotetext{
Vgl. B. Giovannini, « Les menaces sur la planète », CADMOS -- Cahiers trimestriels du Centre Européen de la Culture, Bd. 13, Nr. 49, 1990

${ }^{6}$ Von den zahlreichen Definitionen für nachhaltige Entwicklung soll hier folgende festgehalten werden : « Sustainable development involves devising a social and economic system which ensures [...] that real incomes rise, that educational standards increase, that the health of the nation improves, that the general quality of life is advanced. " Vgl D. Pearce, A. Markandya und E. Barbier, Blueprint for a Green Economy, London, Earthscan, 1992, S. 1-2

Vgl. G. M. Grossmann und A. B. Krueger, Economic Growth and the Environment, Cambridge University Press, 1994
} 
grundlegender Wandel in der Verteilung der finanzellen Mittel statt, wie etwa eine Kürzung der Rüstungsausgaben zugunsten der Umweltpolitik. ${ }^{8}$ Dazu kommt, dass in Wachstumsphasen das politische Klima für umweltpolitische Massnahmen günstiger ist, während in Phasen der Rezession andere Probleme wie etwa die Arbeitslosigkeit die Sorge um die Umwelt verdrängen.

Zwei Bedingungen müssen erfüllt sein, damit eine Synergie zwischen Wirtschaftswachstum und Umweltschutz entstehen kann :

- Eine Palette von rechtlichen, technischen und ökonomischen Massnahmen zur Minimierung der Auswirkungen des Wirtschaftswachstums auf die Umwelt unter Berücksichtigung der jeweiligen Grenzwerte,

- Die Verwendung eines Teils der durch das Wirtschaftswachstum entstandenen Ressourcen zur Finanzierung von Massnahmen gegen die Umweltdegradierung.

Dabei spielen die wirtschaftlichen Instrumente eine besonders wichtige Rolle, da sie einen Ausgleich zwischen den Vorteilen einer Reduktion der durch eine bestimmte Tätigkeit verursachten Umweltbelastung und der dadurch entstehenden Kosten erlauben. So kann beispielsweise bei einer die Luft belastenden industriellen Tätigkeit dank der Internalisierung der Umweltkosten ein Marktpreis berechnet werden, welcher sowohl die von den Unternehmen getragenen privaten Kosten als auch die von der Allgemeinheit getragenen Umweltkosten sowie die Präferenzen der Konsumenten reflektiert.

Die Problematik der Finanzierung des Umweltschutzes kann nicht erörtert werden, ohne auf die Frage nach der Effizienz beim Umgang mit den vorhandenen Mitteln einzugehen. In der Regel sind die ersten Massnahmen die wirksamsten : Eine Investition von 1 Mio. Fr. zur Verringerung des Schadstoffausstosses eines Wärmekraftwerks älteren Datums hat einen weit höheren Wirkungsgrad, als wenn derselbe Betrag in eine moderne Kombianlage investiert wird. Es wäre daher in manchen Bereichen sinnvoller, die Mittel, die zur Bekämpfung der Umweltdegradierung bereitstehen, in den ehemals kommunistischen Staaten und in den Ländern des Südens einzusetzen anstatt in den Industrieländern.

\subsection{UMWELTPOLITIK UND WIRTSCHAFTSWACHSTUM}

Die Massnahmen zur Bekämpfung der Umweltdegradierung lassen sich unter anderem nach ihren Auswirkungen auf das Wirtschaftswachstum unterscheiden. Zum einen gibt es Massnahmen, die sich sowohl in ökologischer als auch in ökonomischer Hinsicht positiv auswirken. Dazu gehören die Streichung gewisser Arten von Subventionen (z.B. Subventionen für Energie, Düngemittel und Pestizide), welche wirtschaftliche Verzerrungen ${ }^{9}$ hervorrufen und gleichzeitig die Verwendung umweltbelastender Substanzen fördern, aber auch Investitionen zur Trinkwasserversorgung oder zum Schutz der Böden. Zum anderen gibt es Massnahmen, die ökonomisch nur dann sinnvoll sind, wenn jene Umweltkosten ein-

${ }^{8}$ Im Vergleich mit anderen Ländern des Südens verfügen die neuen Industrieländer Asiens, die zur Zeit ein starkes Wirtschaftswachstum aufweisen (reale Wachstumsraten von 6 bis 7\%), über ein höheres Potential für Investitionen im Umweltbereich. Zudem profitieren sie von den Erfahrungen der westlichen Länder.

9 Mit « Verzerrung » ist eine schlechte Nutzung produktiver Ressourcen gemeint (natürliches, erarbeitetes und menschliches Kapital), die sich daraus ergibt, dass die Grenzkosten eines Gutes nicht in seinem Preis enthalten sind. 
bezogen werden, die bei ihrer Unterlassung entstehen würden, wie etwa die Einführung einer $\mathrm{CO}_{2}$-Abgabe oder Investitionen in Anlagen zur Emissionsreduktion in mit fossilen Brennstoffen arbeitenden Industriebetrieben oder Wärmekraftwerken. Die letztgenannten Massnahmen stossen auf den grössten Widerstand. In wirtschaftlichen Krisensituationen oder in unterentwickelten Ländern werden derartige Erwägungen meist auf unbestimmte Zeit verschoben.

Obgleich die Herstellung und Vermarktung umweltschondender Technologien für die Industrie ein grosses Zukunftspotential darstellen, haben sich die Erwartungen in diesem Bereich bis heute nicht erfüllt. Grund dafür sind die noch relativ schwach entwickelten und nur auf wenige Regionen begrenzten wirtschaftlichen Impulse und Gesetzgebungen im Umweltschutzbereich.

\subsection{MARKT UND UMWELT}

Die Problematik der Umweltpolitik stellt indirekt die Frage nach dem Kräftegleichgewicht auf den Märkten, anders ausgedrückt nach der « unsichtbaren Hand », die eine stets optimale Verteilung der Ressourcen garantiert. Im Zusammenhang mit der Umwelt können drei Bereiche festgestellt werden, in denen die Märkte versagen :

- Externe Kosten und Nutzen werden in den Preisen nicht berücksichtigt. Anders ausgedrückt : Die Berechnung der Preise erfolgt auf Basis der privaten Kosten und lässt soziale Kosten und Nutzen ausser acht. Dieser Mangel beruht auf den nicht geklärten Eigentumsrechten. Solange Umweltressourcen wie die Meere oder die Atmosphäre frei zugänglich sind und keine Entschädigung für ihre Nutzung entrichtet werden muss, besteht die Gefahr des Missbrauchs.

- Unter gewissen Umständen richtet sich das Verhalten der Wirtschaftssubjekte nach kurzfristigen Erwägungen, während Umweltprobleme oft auf lange Sicht angegangen werden müssen. Dabei kann zwischen den Reaktionen des Marktes und den Bedürfnissen der Umwelt eine Kluft entstehen. Ein Beispiel dafür sind die Investitionen in Forschung und Entwicklung (F\&E) umweltschonender Technologien für die zweite Hälfte des kommenden Jahrhunderts. Offensichtlich liegt dabei der zeitliche Horizont den meisten Unternehmern zu weit entfernt. Möglicherweise müssen jedoch bereits heute beträchtliche Investitionen in F\&E getätigt werden, damit ökonomisch vertretbare Technologien rechtzeitig zur Verfügung stehen.

- Vom ökologischen Standpunkt aus gesehen ist die Einbeziehung der Unsicherheit durch die privaten Wirtschaftssubjekte längst nicht immer ein Vorzug. Zwar kann sich eine risikofeindliche Haltung insofern als vorteilhaft erweisen, als sie zu einer Abkehr von Technologien, Verfahren oder Produkten führen kann, die extrem hohe Umweltkosten verursachen (dem Unternehmer drohen Kosten für die Entschädigung der Opfer). Andererseits kann eine solche Haltung auch dazu führen, dass bestimmte Technologien, die sich gegebenenfalls sowohl in ökonomischer als auch ökologischer Hinsicht als vorteilhaft erweisen könnten, nicht zur Anwendung kommen. So werden beispielsweise Investitionen in F\&E von Technologien, welche ohne fossile Brennstoffe auskommen, nur dann rentabel, wenn die pessimistischen Szenarien bezüglich der Folgen der Erderwärmung tatsächlich eintreffen. 
Damit der Entwicklungsprozess in das Konzept der nachhaltigen Entwicklung eingebunden werden kann, müssen Wege zur Integration der Umweltdimension in die Entwicklungsproblematik gefunden werden. Die folgenden Vorschläge zur Wirtschaftspolitik berücksichtigen einerseits den Aspekt der externen Kosten und andererseits den zeitlichen Horizont und die Ungewissheit.

Hinsichtlich der Einbindung der externen Umweltkosten müssen fünf Grundsätze berücksichtigt werden : ${ }^{10}$.

- Wo immer nötig und machbar, muss Klarheit über die Eigentumsrechte geschaffen werden. Dabei ist zu beachten, dass die Systeme des kollektiven Eigentums - in denen die Gemeinschaft selbst die Zugangs- und Nutzungsbedingungen formuliert - ebenfalls eine rationelle Verwaltung der natürlichen Ressourcen erlauben (Weideland, Wälder, Bewässerungssysteme, Fischereistandorte usw.).

- Die externen Kosten müssen internalisiert werden. Allerdings gibt es in zahlreichen Fällen ausserordentlich grosse Probleme bei der Veranschlagung dieser Kosten. So stellt sich zum Beispiel die Frage, in welcher Höhe die Kosten der Zerstörung der Ozonschicht oder eines nuklearen Störfalls liegen, welche die Ursache für Jahrzehnte später auftretende Erkrankungen wie Krebs oder genetische Fehler sein können. In manchen Fällen wird man einen anderen Ansatz verfolgen müssen, indem nämlich der Preis für umweltbelastende Produkte ausreichend hoch veranschlagt wird, dass der Konsum des Produktes und die damit einhergehende Verschmutzung ein tragbares Volumen nicht überschreiten (« Vermeidungspreis »).

- Projekte mit mehr oder weniger starken Auswirkungen auf die Umwelt sollten einer Art Kosten-Nutzen-Analyse unterzogen werden (oder analog dazu einer Kosten-Wirksamkeits-Analyse). Dies würde es den Verantwortlichen erlauben, die ökologischen und sozialen Auswirkungen eines Projekts zu berücksichtigen, wie z.B. der Verlust von Wohnraum oder besonders wertvoller Biotope beim Bau einer Staumauer, sowie die Ausbildung der Arbeiter oder der Schutz der Böden in einem Projekt zur Rationalisierung der Landwirtschaft einer bestimmten Region.

- Bei der Internalisierung der Kosten und bei Verträglichkeitsstudien müssen sowohl die natürlichen Risiken wie Erdbeben, Vulkanausbrüche oder Überschwemmungen als auch die technologischen Risiken, die besonders für die chemische Industrie und den Nuklearbereich von Bedeutung sind, detailliert einbezogen werden. Bestimmte Katastrophenfälle, deren Wahrscheinlichkeit sehr gering ist, können nämlich schwerwiegende Konsequenzen für die Umwelt und die sozioökonomische Entwicklung einer Region nach sich ziehen. Man erinnere sich nur an den Chemieunfall in Bhopal in Indien, der 1984 2'500 Todesopfer forderte und Tausende von Verletzten hinterliess, oder an das Erdbeben in El Salvador im Jahre 1986, welches Verluste von rund 25\% des BIP und $40 \%$ der Aussenverschuldung des Landes verursachte. ${ }^{11}$

${ }^{10}$ Vgl. P.S. Dasgupta und G.M. Heal, Economic Theory and Exhaustible Resources, Cambridge University Press, 1979 ; OCDE, Gérer l'environnement : le rôle des instruments économiques, Paris, OCDE, 1994; sowie R. Sugden und A. Williams, The Principles of Practical Cost-Benefit Analysis, Oxford University Press, 1978.

${ }^{11} \mathrm{Vgl}$. C. Clarke und M. Munasinghe (Hrsg.), Disaster Prevention for Sustainable Development, Washington, US National Academy of Sciences, Weltbank, im Druck. 
- Generell muss in diesem Zusammenhang die Bedeutung bestimmter Analysemethoden unterstrichen werden, insbesondere die Umweltbuchhaltung in den Unternehmen, Analysen des Lebenszyklus der Güter sowie die Einbeziehung der Umwelt in die Staatsrechnung.

Im Zusammenhang mit Problemen bezüglich des zeitlichen Horizonts und des Unsicherheitsmanagements sollen hier lediglich die Aufgaben der Umweltpolitik aufgezeigt werden. ${ }^{12}$ Sie muss ein Paket von Massnahmen bereitstellen, welche :

- flexibel genug sind, um neuen Erkenntnissen und der Verringerung der Unsicherheitsmarge angepasst zu werden ;

- ausgeglichen genug sind (oder, anders ausgedrückt, in einem vernünftigen Verhältnis zu den erwarteten Gewinnen stehen), um zu verhindern, dass zuviel Geld ausgegeben und Ressourcen verschwendet werden, oder dass zu wenig ausgegeben wird und eines Tages das Handlungsdefizit teuer bezahlt werden muss ;

- effizient genug sind, um irreversible Prozesse zu vermeiden.

Diese Ausführungen zeigen, wie komplex der Entscheidungsprozess angesichts einer ungewissen Zukunft sein kann. Einerseits muss zwischen Kurzfristigkeit und Langfristigkeit sowie zwischen mehreren möglichen Naturzuständen abgewogen werden, andererseits muss man sich stets vor Augen halten, dass es sich in Umweltbelangen oft um irreversible Phänomene handelt. Ein treffendes Beispiel für dieses Problem sind die Schwierigkeiten, die sich bei der Erarbeitung von Strategien zur Bekämpfung des Treibhauseffekts stellen.

Tabelle 1

Fördernde und regulierende Massnahmen im Bereich des Umweltschutzes ${ }^{13}$

\begin{tabular}{|c|c|c|c|}
\hline \multicolumn{2}{|c|}{ Art der Massnahmen } & \multicolumn{2}{|c|}{ Betroffene Variable } \\
\hline & Preis & Menge & Technologie \\
\hline \multicolumn{4}{|l|}{ Fördernd } \\
\hline Direkt & $\begin{array}{l}\text { Abgaben auf Abwässer, } \\
\text { Abholzungssteuer, } \\
\text { Hinterlegungen }\end{array}$ & $\begin{array}{l}\text { Handelbare Emissions- } \\
\text { rechte, handelbare } \\
\text { Fischereirechte }\end{array}$ & $\begin{array}{l}\text { Abgaben auf vor- } \\
\text { aussichtliche } \\
\text { Emissionen }\end{array}$ \\
\hline Indirekt & $\begin{array}{l}\text { Treibstoffsteuern, } \\
\text { Ökosteuern }\end{array}$ & $\begin{array}{l}\text { Handelbare Produk- } \\
\text { tions- oder Produktions- } \\
\text { mittelkontingente }\end{array}$ & $\begin{array}{l}\text { Subventionen für } \\
\text { F\&E und energie- } \\
\text { parende Technologien }\end{array}$ \\
\hline \multicolumn{4}{|c|}{ Regulierend } \\
\hline Direkt & - & $\begin{array}{l}\text { Emissionnormen, } \\
\text { Abholzungsquoten }\end{array}$ & $\begin{array}{l}\text { Zwingende techni- } \\
\text { sche Normen }\end{array}$ \\
\hline Indirekt & - & $\begin{array}{l}\text { Zonung, Verbot oder } \\
\text { Kontingentierung der } \\
\text { Produkte oder der } \\
\text { Produktionsmittel }\end{array}$ & $\begin{array}{l}\text { Leistungsnormen } \\
\text { für Produktions- } \\
\text { mittel und Verfahren }\end{array}$ \\
\hline
\end{tabular}

${ }^{12}$ Vgl. R.S. Pyndick, « Irreversibility, Uncertainty and Investment », Journal of Economic Literature, Bd. 29, Nr. 3, 1991.

${ }^{13}$ Quelle : Weltbank, ibidem, 1992, S. 77. 
In Tabelle 1 sind einige wirtschaftliche und rechtliche Massnahmen der Umweltpolitik aufgeführt, die in den Industrieländern zur Anwendung kommen. Zu erwähnen ist ausserdem, dass in bestimmten Bereichen wie z.B. im Gesundheitswesen dank Informations- und Aufklärungsarbeit gute Ergebnisse erzielt werden können, ohne dass auf derartige Massnahmen zurückgegriffen werden muss. In bezug auf die Tabelle scheinen folgende Erklärungen angezeigt :

- Es wird zwischen fördernden, auf die Mechanismen des Marktes wirkenden Massnahmen (« Marktanreize ») und regulierenden, eher dirigistischen Massnahmen («Steuerungen und Vorschriften ») unterschieden. ${ }^{14}$ Die letztgenannten werden unumgänglich, wenn es sich um ausserordentlich schwerwiegende Gefahren für die Umwelt wie Giftmüll, radioaktive Substanzen usw. handelt. Chronologisch gesehen gingen die regulierenden Massnahmen den fördernden Massnahmen voraus. Bis vor kurzem wurde versucht, die Umweltverschmutzung hauptsächlich mit regulierenden Massnahmen in den Griff zu bekommen. Erst in letzter Zeit kommen auch fördernde Massnahmen zum Einsatz. Eine der Schwierigkeiten der Umweltpolitik besteht darin, ein Gleichgewicht zwischen den beiden Massnahmenkategorien zu finden.

- Bevor sie zur Anwendung kommen können, müssen sämtliche Massnahmen sowohl im Hinblick auf die Verteilung der Mittel und ihre Wirkungen auf die Umwelt, als auch unter dem Gesichtspunkt ihrer Auswirkungen auf die Gesellschaft und insbesondere auf deren schwächste Mitglieder untersucht werden (unter Umständen müssen begleitende Massnahmen in Betracht gezogen werden, um die Benachteiligung in Grenzen zu halten).

- Eine Harmonisierung der Umweltpolitiken auf internationaler Ebene ist von grosser Wichtigkeit, denn nur so kann verhindert werden, dass risikoreiche und verschmutzende Aktivitäten in weniger anspruchsvolle Länder transferiert werden.

- Schliesslich darf der Kampf gegen die Umweltkriminalität (z.B. die Einrichtung wilder Deponien für gefährliche Abfälle), die eine grosse Gefahr für den Erfolg der Umweltpolitik darstellt, nicht ausser acht gelassen werden.

\section{ENTWICKLUNGSHILFE UND UMWELT}

Im folgenden werden einige Instrumente diskutiert, die es erlauben, die ökologischen Aspekte in die Entwicklungshilfe der Industrieländer, darunter der Schweiz, und der im Entwicklungsbereich tätigen internationalen Organe einzubeziehen. Dabei beschränken wir uns auf die relativ direkt wirkenden Instrumente. Instrumente mit indirekter Wirkung auf die Umwelt wie etwa jene aus dem Bereich der Erziehung, des Gesundheitswesens und der Bevölkerungspolitik werden hier nicht besprochen.

\subsection{SCHWERPUNKT ÖKOLOGIE IN DER ENTWICKLUNGSHILFE}

Sowohl auf lokaler als auch auf globaler Ebene können ökologische Zielsetzungen zum Teil dadurch erreicht werden, dass ökologische Aspekte in bestehende

\footnotetext{
${ }^{14}$ Regulierende Massnahmen im weiteren Sinne könnten die Definition der Eigentumsrechte einschliessen.
} 
bilaterale oder multilaterale Entwicklungshilfeprogramme integriert oder vermehrt berücksichtigt werden. So kann die öffentliche Entwicklungshilfe etwa spezifische Umweltschutzprojekte fördern. Wie bereits ausführlich dargelegt wurde, kann die Verbesserung der Umweltbedingungen das Wirtschaftswachstum der Entwicklungsländer positiv beeinflussen und sich somit doppelt auszahlen. Ausserdem messen mehrere von der Schweiz finanzierte Entwicklungsprojekte der Umwelt besonderes Gewicht bei. Als Beispiele für solche Projekte aus dem landwirtschaftlichen Bereich sind zu erwähnen : ${ }^{15}$

- das forstwirtschaftliche Programm zur nachhaltigen Bewirtschaftung der Wälder Madagaskars,

- das Pflanzen von Bäumen in den Reisfeldern im Nordosten Bangladeshs,

- das Programm zur Verbesserung der Fruchtbarkeit der Böden in den ariden Gebieten und den Bergregionen Nepals,

- die biologische Bekämpfung von Parasitenbefall in Maniokpflanzungen in Afrika, und

- die Produktionsförderung in den von Nomaden bewohnten Trockengebieten des Sahel.

In den kommenden Jahren dürfte die Zahl der Entwicklungsprojekte mit direkter Wirkung auf die Umwelt weiter ansteigen.

Wird jedoch in naher Zukunft das Volumen der öffentlichen Entwicklungshilfe nicht massiv erhöht, was angesichts der gegenwärtigen Finanzkrise in den meisten Industrieländern einschliesslich der Schweiz unwahrscheinlich sein dürfte, besteht die Gefahr, dass andere, mindestens ebenso wichtige Entwicklungsprogramme nicht mehr mit den heute budgetierten Beträgen finanziert werden können. Deshalb ist der Handlungspielraum zur Einbeziehung von Umweltprojekten in bestehende Entwicklungsprogramme für die Länder des Südens beschränkt. Hingegen dürfte es weitaus einfacher sein, bei der Prüfung neuer Projekte ökologische Gesichtspunkte einzubeziehen. Der Gedanke, externe Aspekte in die Kosten-Nutzen-Analyse zu integrieren, bevor über die Finanzierung des Projekts entschieden wird, findet langsam seinen Weg in die Entwicklungspolitik der westlichen Industrieländer. Leider hat sich in der Schweiz diese Art von Nutzwert-Analysen noch nicht durchgesetzt.

Seit den achziger Jahren tragen internationale Organe wie die Weltbank derartigen Überlegungen bei der Entscheidung über die Finanzierung von Projekten in Entwicklungsländern verstärkt Rechnung. ${ }^{16}$ Im Anschluss an eine Reihe stark kritisierter Kreditvergabeoperationen, wie z.B. für das Projekt Polonoroeste in Brasilien, für das Projekt zur Förderung der Viehzucht in Botswana und für das Transmigrationsprojekt in Indonesien, beschloss die Weltbank 1987 eine grundlegende Revision ihrer Politik, die ökologischen Aspekten künftig grosse Bedeutung beimessen sollte. Es wurden neue Verfahren ausgearbeitet, in erster Linie die Erstellung länderspezifischer Rapporte über die Umweltproblematik, die nötigenfalls anhand von Studien auf regionaler Ebene ergänzt werden können. Die Ergebnisse dieser Untersuchungen werden in die landesspezifischen Sektoren- und Wirtschaftsanalysen integriert, welche der Weltbank als Grundlage für

\footnotetext{
${ }^{15} \mathrm{Vgl}$. dazu die Botschaft vom 20.4.1994 über die Weiterführung der technischen Zusammenarbeit und der Finanzhilfe zugunsten von Entwicklungsländern, BB Bd. 146, 1994/II, S. 941 ff.

${ }^{16} \mathrm{Vgl}$. J. Warford und Z. Partow, « Die Umweltpolitik der Weltbank », Finanzierung und Entwicklung, IWF/IBRD, Dezember 1989.
} 
die Kreditvergabe und die Festsetzung der wirtschaftspolitischen Prioritäten des jeweiligen Landes dienen.

Ein weiteres Instrument, das der Völkergemeinschaft bei der Verfolgung globaler ökologischer Zielsetzungen in den Entwicklungsländern zur Verfügung steht,.sind die Programme zur Strukturanpassung mit Hilfe von Strukturanpassungsfazilitäten (SAF). Angesichts der reellen Deregulierungen auf den Kapital- und Geldmärkten und im Budgetbereich, sowie der Schuldenkrise und der Schwierigkeiten beim Schuldendienst haben zahlreiche Entwicklungsländer Afrikas, Lateinamerikas und Osteuropas begonnen, vom Internationalen Währungsfonds ausgearbeitete Programme zur Wiederherstellung ihres Aussenhandels-, Budget- und Währungsgleichgewichts zu verfolgen. Diese Programme haben eine mehr oder weniger direkte Wirkung auf die Umwelt. Zum einen können diese Auswirkungen durchaus positiver, wenn nicht unmittelbarer Natur sein, je nachdem, welche Verbesserungen in Bereichen wie dem Gesundheitswesen, der Erziehung oder dem Bevölkerungswachstum erreicht werden. Zudem zielen die meisten dieser Programme auf die Wiederherstellung der «Preiswahrheit» ab, so dass insbesondere dank der Verringerung bzw. Abschaffung staatlicher Subventionen auf die Energiepreise auch direktere ökologische Wirkungen erzielt werden können.

$\mathrm{Zu}$ bemerken ist allerdings, dass die Umsetzung dieser Programme oft schwierig ist und unter Umständen verhängnisvolle Auswirkungen auf die Umwelt haben kann. So haben Kritiker darauf hingewiesen, dass die Programme aufgrund der Exportförderungen einen verstärkten Abbau natürlicher Ressourcen wie Tropenhölzer bewirken können, der mit dem Prinzip der nachhaltigen Entwicklung nicht vereinbar ist.

\subsection{INTERNATIONALE ABKOMMEN}

Internationale Verhandlungen sind ein relativ « kostengünstiges » Mittel, welches es den Industrieländern und den internationalen Organisationen erlaubt, im Umweltbereich Einfluss zu nehmen. Das erste Umweltschutzabkommen von globaler Bedeutung war das Protokoll von Montreal aus dem Jahre 1987 über Substanzen, die die Ozonschicht gefährden. ${ }^{17}$ Die Vertragsparteien des Protokolls von Montreal verpflichteten sich einerseits, die Herstellung chemischer Verbindungen, die zum Abbau der Ozonschicht in der Stratosphäre beitragen, bis zum Jahr 2000 progressiv um die Hälfte zu reduzieren, und andererseits, die weitere Verbreitung der Treibgase auf den Märkten zu unterbinden. Nach Bekanntwerden neuer beunruhigender Tatsachen über das Ausmass der Zerstörung der Ozonschicht und der Geschwindigkeit dieses Prozesses erfuhr das Protokoll von Montreal im Juni 1990 eine tiefgreifende Revision. Neu ist ein totales Verbot der Fluorkohlenwasserstoffe, der Halogen- und Tetrachlorkohlenstoffe bis zum Jahr 2000 vorgesehen, einige weitere Substanzen mit ähnlich zerstörerischer Wirkung sollen bis 2005 verboten werden. Begleitend zu dieser Revision wurde ein OzonFonds eingerichtet, der es Entwicklungsländern erlauben soll, den Verbindlichkeiten nachzukommen, die sich für sie aus dem Abkommen ergeben.

${ }^{17}$ Vgl. Bundesamt für Umweltschutz, Wald und Landschaft, Zur Lage der Umwelt in der Schweiz 1993, BUWAL, 1994. 
Das wichtigste Ereignis der letzten Jahre in diesem Zusammenhang war die UNCED, die 1992 in Rio stattfand. Da jedoch bezüglich der Fristen für zu ergreifende Massnahmen oder der Verstärkung der Hilfsprogramme keinerlei konkrete Vereinbarungen getroffen werden konnten, wird das Ergebnis der Konferenz, gemessen an den Erwartungen, heute als relativ enttäuschend bezeichnet. Trotz allem wurden an der Konferenz verschiedene Schlussdokumente verabschiedet und zwei Abkommen unterzeichnet. Die Deklaration von Rio über Umwelt und Entwicklung, die Agenda 21 und die Rahmenprinzipien über den Waldschutz sind rechtlich nicht bindend. Die Klimaschutzkonvention und die Artenschutzkonvention hingegen ziehen rechtliche Verpflichtungen nach sich und müssen vor ihrem Inkrafttreten von den Unterzeichnerstaaten ratifiziert werden.

Die Deklaration von Rio ist eine kurze Darlegung von Grundprinzipien, die das menschliche Verhalten gegenüber der Umwelt regeln und die Rechte und Pflichten der Staaten, der Gemeinschaften und des Einzelnen definieren. Sie ist eine weniger ambitiöse Version der ursprünglich vorgesehenen Erdcharta. Die Idee einer Erdcharta, der ähnliche Bedeutung hätte zukommen sollen wie der Allgemeinen Erklärung der Menschenrechte, wurde von der Schweiz mitgetragen, konnte sich aber am Gipfel von Rio aufgrund der Divergenzen zwischen den Industrie- und den Entwicklungsländern nicht durchsetzen. Die Industrieländer wollten der Ökologie und der gemeinsamen Verantwortung gegenüber der globalen Umwelt grössere Bedeutung beimessen, während die Entwicklungsländer der Entwicklungsproblematik den Vorrang geben wollten. Bei der Agenda 21 handelt es sich um ein ambitiöses Dokument mit einem 800seitigen, über 100 Themengebiete umfassenden Aktionsplan für das 21. Jahrhundert. In den ersten Kapiteln werden soziale und ökonomische Aspekte der nachhaltigen Entwicklung erörtert, insbesondere Strategien zur Bekämpfung der Armut und zur Förderung der Gesundheit der Bevölkerung. Der grösste Teil des Dokuments jedoch befasst sich mit spezifischen Umweltproblemen. Zu erwähnen ist, dass sich jeder Unterzeichnerstaat zur Umsetzung der Agenda 21 auf nationaler Ebene verpflichtet hat. In der Schweiz wurde ein interdepartementaler Ausschuss mit der Erarbeitung eines nationalen Aktionsplans betraut. Bei den Rahmenprinzipien über den Waldschutz schliesslich handelt es sich um eine einfache Empfehlung zur Beschleunigung der Programme zur Aufforstung und zur Bewahrung der Wälder, welche ausserdem die Rechte der Bevölkerung und der Empfängerstaaten bei der Bewirtschaftung der Wälder verstärkt berücksichtigen soll.

Die rechtlich bindende Klimaschutzkonvention ist eine der wichtigsten Errungenschaften der UNCED. Sie hat zum Ziel, den Treibhauseffekt unter Kontrolle $\mathrm{zu}$ bekommen, indem die Konzentration von Treibhausgasen in der Luft, vor allem von Kohlendioxid, stabilisiert werden soll. Die Industrieländer sind sich darin einig, dass eine Senkung der Emissionen auf das Niveau von 1990 bis zum Jahr 2000 erstrebenswert ist. Die Konvention wurde von 120 Staaten, darunter der Schweiz, ratifiziert und trat am 21. März 1994 in Kraft. Die erste Sitzung der Konferenz der Unterzeichnerstaaten fand im März und April 1995 in Berlin statt. Obgleich sämtliche vertretenen Staaten darin übereinstimmten, dass das für das Jahr 2000 gesetzte Ziel nur einen Meilenstein darstellt, konnte über die danach $\mathrm{zu}$ ergreifenden Massnahmen keine Einigung erzielt werden. In einer ersten Phase besteht aber die Möglichkeit, die Emissionen auf freiwilliger Basis gemeinsam weiter zu senken. Während der Debatten kamen mehrere wichtige Fragen der Nord-Süd-Problematik zur Sprache, insbesondere in bezug auf die 
jeweilige Verantwortung der Industrie- bzw. Entwicklungsländer sowie den Transfer umweltverträglicherer Technologien. Allerdings stellt keine der beiden Ländergruppen einen geschlossenen Block dar. So haben sich gewisse Industrieländer, darunter die Schweiz, durchaus bereit erklärt, in diesem Bereich weiter zu gehen als beispielsweise die USA. In der Gruppe der Entwicklungsländer finden sich auf der einen Seite die kleinen Inselstaaten, die strengere und radikalere Massnahmen befürworten, und auf der anderen Seite die erdölexportierenden Staaten, die sich jeglicher Einschränkung der Ausbeutung fossiler Energiequellen widersetzen.

Die Artenschutzkonvention ist die zweite Errungenschaft der UNCED. Gemäss diesem Abkommen sind die Staaten verpflichtet, gesetzliche Massnahmen zum Schutz ihrer biologischen Ressourcen auszuarbeiten. Sie überträgt den Nationen die rechtliche Verantwortung für das Verhalten ihrer privaten Unternehmen in anderen Staaten und empfiehlt, den Entwicklungsländern als Gegenzug für die Ausfuhr von Genmaterial technische Unterstützung und Entschädigungszahlungen zukommen zu lassen. Allerdings enthält die Konvention keine eindeutigen Hinweise über die Durchsetzung dieser Prinzipien. Höchstwahrscheinlich wird ein Netz von bilateralen Verträgen oder Handelsabkommen diese Lücke schliessen. ${ }^{18}$ Die Konvention wurde in Rio von 156 Ländern einschliesslich der Schweiz unterzeichnet.

Die zeitaufwendigen Verfahren und die Fragilität ihrer Ergebnisse sind ein grosser Nachteil der internationalen Verhandlungen im Umweltbereich. ${ }^{19}$ Die Erfahrung hat gezeigt, dass Verhandlungen auf diesem Gebiet sowohl auf regionaler als auch auf internationaler Ebene äusserst langwierig und komplex sind. Das Protokoll von Montreal, dessen Abschluss dank dem Druck der Wissenschaftler und der Bereitschaft der Unternehmen beschleunigt wurde, stellt hier eine Ausnahme dar. Nicht selten verstreichen ein oder zwei Jahrzehnte bis zum Abschluss eines Abkommens oder Vertrags, wie etwa beim Seerecht, der Verhandlungen über die grossen Flüsse oder derjenigen über den Klima- und den Artenschutz. Die Ursache für diese Langwierigkeit liegt einerseits in der Komplexität der Materie, was dazu führen kann, dass während der Verhandlungen Fragen ohne direkten Bezug zur Umwelt auftauchen, andererseits aber auch im kulturell bedingten Verhalten der Unterhändler selbst, sowie in den hauptsächlich finanziellen Möglichkeiten und Grenzen der Durchsetzung von Konventionen und Verträgen. Daraus folgt, dass der wissenschaftlichen Erforschung des Verhandlungsprozesses sowie der Ausbildung der Unterhändler der Entwicklungsländer im Bereich der Angewandten Sozialwissenschaften in bezug auf die Umweltund Entwicklungsproblematik ein äusserst hoher Stellenwert zukommen muss. Unter anderem geht es darum, das Verständnis der massgebenden völkerrechtlichen Schriften aus dem technischen, rechtlichen und finanziellen Bereich zu fördern und auf diese Weise eine Beschleunigung des Verhandlungsprozesses zu erwirken. $\mathrm{Zu}$ erwähnen ist in diesem Zusammenhang, dass die teilweise vom Bund unterstützte Académie internationale de l'environnement in Genf die Bedeutung dieses Aspektes erkannt hat und der Ausbildung von Unterhändlern aus den Ländern des Südens grosses Gewicht beimisst.

\footnotetext{
${ }^{18}$ Ein Beispiel dafür wäre das Abkommen, welches zwischen dem multinationalen Pharmakonzern Merck und dem Nationalen Institut für Artenschutz von Costa Rica geschlossen wurde. Vgl. dazu auch « Biodiversité au Costa Rica INBIO veritas », Solidaire, Nr. 121, Februar 1994.

${ }^{19} \mathrm{Vgl}$. A. Banachenhou, « Environnement et développement », Revue Tiers Monde, Bd. 23, Nr. 130, April-Juni 1992.
} 


\subsection{HANDELSPOLITIK}

Die Auswirkungen des internationalen Handels auf die Umwelt können unterschiedlich sein. ${ }^{20}$ Erstens kann er ein Anreiz sein, die Produktion aus ökologisch gefährdeten Zonen in Gebiete zu verlegen, in denen die Produktivität längerfristig gesichert ist, und umgekehrt. Zweitens bewirkt die Liberalisierung strukturelle und volumenmässige Veränderungen im Konsum, in der Produktion und im weltweiten Einkommen, was für die Umwelt weit mehr als lediglich eine Verschiebung des Konsums und der Produktion von einem Land ins andere zur Folge haben kann. Drittens übt der Handel einen Einfluss auf die wirtschaftliche Entwicklung aus und schafft neue Lücken für einen gewinnbringenden Einsatz der Produktionsfaktoren. So stellen beispielsweise die Agrarexporte für zahlreiche Länder wichtige Devisenquellen dar.

Es muss hinzugefügt werden, dass ein Einkommenszuwachs, unter anderem aufgrund der erhöhten Mobilität, einen Anstieg der Nachfrage nach natürlichen Ressourcen zur Folge hat, der sich negativ auf die Umwelt auswirkt. Andererseits kann sich dieser Einkommenszuwachs auch in höheren Anforderungen an die Umweltqualität niederschlagen. Parallel dazu lassen sich Investitionen zur Bewahrung der natürlichen Ressourcen einfacher realisieren und werden von der Bevölkerung positiver aufgenommen. Geht man ausserdem davon aus, dass der Einkommenszuwachs mit einem Rückgang des Bevölkerungswachstums einhergeht, so verringert sich die Belastung der natürlichen Ressourcen. Bei gleichzeitiger Verbesserung der Einkommens- und der Arbeitslage steigt die Zahl der Möglichkeiten, und die ländliche Bevölkerung wird nicht weiter in ökologisch gefährdete Gebiete wie etwa Steilhänge abgedrängt.

Die äusserst komplexen Beziehungen zwischen Handel, Entwicklung und Umwelt werden somit offensichtlich. Während der ersten Phase der GATT-Verhandlungen (Uruguay-Runde) wurden mehrere Studien über die ökologischen Folgen der Liberalisierung der multilateralen Handelsbeziehungen erstellt, wobei der Schwerpunkt auf die Landwirtschaft und die Ausbeutung natürlicher Rohstoffe gelegt wurde. ${ }^{21}$ Aus all diesen Analysen lässt sich schliessen, dass sich eine Reduktion der Verzerrungen auf den Agrarmärkten in entwickelten Volkswirtschaften positiv auf die Umwelt auswirken kann, sofern diese Reduktion über eine Abkehr von intensiven Bewirtschaftungsmethoden, begleitet von einer Verringerung des Einsatzes von Düngemitteln und Pestiziden, über eine anderweitige Nutzung der Böden, sowie über eine Verkleinerung der Landwirtschaftsbetriebe oder die Einstellung eines Teils der Agrarproduktion erreicht wird. In den südlichen Volkswirtschaften hingegen dürfte die Liberalisierung der Handelsbeziehungen eher das Gegenteil bewirken. Sollten sich die Handelsbedingungen verbessern, so bedeutet dies für die Landwirtschaft einen Übergang zu intensiveren Anbaumethoden (Mechanisierung, Einsatz von Düngemitteln und Pestiziden) mit all ihren Folgen im Hinblick auf Nutzung und Verschmutzung des Wassers, Erosionsgefahr und Ausdehnung der Anbauflächen. Ohne diese Schlussfolgerungen auf alle von der Liberalisierung betroffene Bereiche ausdehnen zu wollen, scheint es uns doch legitim, bereits jetzt Zweifel an den vorteilhaften Aus-

${ }^{20} \mathrm{Vgl}$. FAO, « Le commerce agricole à l'aube d'une ère nouvelle », Rapport 1995, FAO, Rom, 1995.

${ }^{21}$ Für eine erschöpfende Liste dieser Studien vgl. J. Beghin, D. Roland-Holst und D. van der Mensbrugghe, « Etude de la relation entre commerce et environnement : dimensions globales ", Revue économique de l'OCDE, $\mathrm{Nr}$. 23, Winter 1994. 
wirkungen des Handels auf die Umwelt in den Entwicklungsländern zu äussern.

Trotz allem stellen die Möglichkeiten der Handelspolitik ein kostengünstiges Instrumentarium dar, um die Entwicklungsländer dazu zu bewegen, weniger umweltschädliche Konsumgewohnheiten und Produktionsmethoden anzunehmen. Es geht darum, in diesen Ländern die als umweltfreundlicher geltenden Normen des Westens einzuführen. Dies kann auf mehr oder weniger sanfte Art geschehen. Zum einen können die Entwicklungsländer dazu ermutigt werden, anhand einer einfachen Modifikation ihrer Exportbedingungen die Umweltkosten bis zu einem bestimmten Grad zu internalisieren (siehe weiter unten). Aber der Welthandel kann auch direktere positive Auswirkungen auf die Umwelt haben. Einer kürzlich von der Umwelt- und Entwicklungskonferenz der Vereinten Nationen (UNCED) erstellten Studie zufolge tragen die Handelsbeziehungen zu einer Verbreitung umweltfreundlicher Standards bei. ${ }^{22}$ So stellen beispielsweise die ökologischen Anforderungen der grossen internationalen Märkte einen Anreiz für die Entwicklungsländer dar, ihre Produktionsnormen und gesetzlichen Bestimmungen über den inländischen Markt zu verbessern.

Aber auch durch Ausübung von Druck auf die Entwicklungsländer kann die Handelspolitik auf den Umweltschutz Einfluss nehmen. So sehen die GATTBestimmungen vor, dass jedes Land seine eigenen ökologischen Standards festsetzen kann (Art. XX) ${ }^{23}$ Die einzelnen Länder haben somit die Möglichkeit, die Einfuhr von Gütern zu verbieten, die nicht einer bestimmten Umweltnorm entsprechen. So können beispielsweise Lebensmittel mit Pestizidrückständen oder Spielzeuge, die mit toxinhaltiger Farbe bemalt sind, von den nationalen Märkten ferngehalten werden. Bei der Durchsetzung solcher nationaler Standards müssen die betreffenden Länder lediglich nachweisen, dass einheimische und ausländische Produkte gleich behandelt werden. Allerdings stellt sich hier die Frage nach der Grenze zwischen Umweltschutz und verstecktem Protektionismus. In den vergangenen Jahren sind mehrere aufschlussreiche Streitigkeiten in bezug auf diese ökologischen Standards zutage getreten. Beispiele für die zahlreichen handelspolitischen Meinungsverschiedenheiten im Zusammenhang mit dem Umweltschutz wären jene bezüglich der Vorschriften über die Verpackung von Getränken oder über den Minimalanteil von Recyclingpapier in gewissen Erzeugnissen der Papierindustrie. ${ }^{24}$

$\mathrm{Zu}$ erwähnen ist ausserdem, dass die GATT-Bestimmungen bisher nur abwärts, d.h. auf die Erzeugnisse selbst, und noch nicht aufwärts bzw. auf die Herstellungsverfahren anwendbar sind. Allerdings befassen sich die Vertreter des Umweltschutzes auch vermehrt mit den externen Aspekten der Herstellung wie der Verschmutzung, dem effizienten Einsatz der Energien und der Entsorgung von Abfällen. Darüber hinaus stellt sich für die Industrieländer die Frage nach dem Schutz vor dem sogenannten «Öko-Dumping », welches Entwicklungsländern mit weniger strengen Produktionsnormen einen unfairen Wettbewerbsvorteil verschafft. Angesichts der zunehmenden Verbreitung des «Öko-Labels» und der Gefahr, dass dieses eine Barriere für Importe aus Entwicklungsländern

${ }^{22}$ UNCED, Sustainable Development : Trade and Environment - The Impact of Environment-related Policies on Export Competitiveness and Market Access, TD/B/41(1)4, 1994.

${ }^{23}$ Vgl. P. Uimonen, «Trade Rules and Environmental Controversies During the Uruguay Round and Beyond ", The World Economy, Bd. 18, Nr. 1, 1995.

${ }^{24}$ Vgl. S. Vaughan, «Trade and Environment : Building a Revolutionary Framework ", Industry and Environment, Januar-März 1994. 
darstellen könnte, scheint eine internationale Harmonisierung der Produktionsnormen unabdingbar und umso dringlicher, als immer mehr solcher Labels ohne Kontrolle durch die zuständigen nationalen Behörden geschaffen werden. In der Schweiz beispielsweise steht allein das Bourgeon-Label für biologische Produkte unter dem Schutz der Bundesbehörden. ${ }^{25}$ Die Festlegung von Normen kann sich für jene Länder negativ auswirken, die aufgrund ihres Entwicklungsrückstandes oder ihres Mangels an Know-How und technischen Möglichkeiten nicht in der Lage sind, diese zu respektieren. Es ist deshalb ausserordentlich wichtig, die tatsächlichen Verhältnisse der Entwicklungs- und Schwellenländer bei der Ausarbeitung dieser Normen zu berücksichtigen, indem man sie in den Prozess einbezieht. Mit dieser Aufgabe könnte beispielsweise die WTO betraut werden.

Auch in der Schweiz steigt das Bewusstsein der Konsumenten für die Zusammenhänge zwischen Handel, Entwicklung und Umwelt stetig und und zeigt sich im verstärkten Bedürfnis, sich über die Produktionsbedingungen von Gütern aus Entwicklungsländern zu informieren. ${ }^{26}$ Aus diesem Grund beteiligt sich die Eidgenossenschaft an den multilateralen Bestrebungen im Hinblick auf eine möglichst umfassende Transparenz über Herkunft und Produktionsbedingungen bestimmter ökologisch bedenklicher Produkte wie Tropenhölzer. In diesem Zusammenhang erwägt sie auch die Einführung fakultativer Labels, beispielsweise für Tropenhölzer aus nachhaltig bewirtschafteten Wäldern. Ausserdem bemüht sich die Schweiz, bei der Einführung nationaler Umweltschutzbestimmungen und bei Handelsbeschränkungen sämtliche Massnahmen zu vermeiden, die negative wirtschaftliche Auswirkungen auf die Entwicklungsländer haben oder deren Umwelt längerfristig beeinträchtigen könnten. Die Schweiz ist sich bewusst, dass jene Entwicklungsländer, die aus finanziellen und technischen Gründen nicht in der Lage sind, die entsprechenden Umweltschutznormen einzuhalten, Unterstützung zur Verbesserung ihrer Produktions- und Verarbeitungsnormen erhalten sollten. In einer kürzlich erschienenen Studie über die Auswirkungen der Uruguay-Runde auf die Entwicklungsländer werden einige konkrete Massnahmen vorgestellt, die der Eidgenossenschaft im Hinblick auf die Verwaltung der Öko-Label und zur Vorbeugung gegen den Öko-Protektionismus zur Verfügung stehen. ${ }^{27}$

\subsection{INTERNATIONALE ROHSTOFFABKOMMEN}

Die Schädigung der Ökosysteme in den Entwicklungsländern stehen oft im Zusammenhang mit dem internationalen Rohstoffhandel. In den meisten Herkunftsländern fehlen die finanziellen Mittel, die zum Schutz und zur Wiederherstellung des Umweltgleichgewichts nötig wären. Aufgrund der starken Konkurrenz auf den internationalen Rohstoffmärkten ist es nicht möglich, den Preis um eine bestimmte Summe zu erhöhen, die die Umstellung auf umweltfreundlichere Produktionsmethoden erlauben würde. Auf den nichtorganisierten Rohstoffmärkten ist eine Integration der externen Umweltkosten schlechthin unmöglich.

\footnotetext{
${ }^{25} \mathrm{Vgl}$. M. Egger und J. Forster, « Les mesures de coopération au dévoloppement », in : P. Dembinski, J. Forster und J. de Melo (Hrsg.), Effets de l'Uruguay Round sur les pays en développement, Mandat de l'OFAEE, Genf, 1994 (Bd. 4, Abschnitt B)

${ }^{26} \mathrm{Vgl}$. Bericht des Bundesrates über die Nord-Süd-Beziehungen der Schweiz in den 90er Jahren (Leitbild Nord-Süd) vom 7. März 1994, in Jahrbuch Schweiz-Dritte Welt 1995, S. 233.

${ }^{27}$ Vgl. P. Dembinski et al., op. cit., 1995.
} 
Die internationalen Rohstoffabkommen gehören zu den wenigen internationalen Instanzen, die auf globaler Ebene den Preis, die Produktion und den Konsum gleichzeitig steuern können. Grundsätzlich besteht die Möglichkeit, diese Abkommen zu modifizieren und somit einen Zuschlag auf die Preise zu erheben, der den Übergang zu umweltfreundlichen Produktionsmethoden ermöglichen würde. ${ }^{28}$ Dieser Zuschlag könnte als Ausfuhrsteuer oder in Form einer zusätzlichen Einfuhrsteuer erhoben werden. Die erstgenannte Möglichkeit würde zusätzliche Vorkehrungen sowie eine Revisionsbürokratie voraussetzen, um zu verhindern, dass sich manche Länder ihren Verpflichtungen entziehen könnten und die am wenigsten entwickelten Länder ihre Importe zu teuer bezahlen müssten. Die zweite Möglichkeit, also die Erhebung eines zusätzlichen Importzolls bei der Einfuhr in die Verbraucherländer, scheint besser geeignet. Auf diese Weise könnten die an die Produktion und den Verbrauch gekoppelten Finanzmittel einem internationalen Fonds zugeführt werden, aus welchem Projekte zum Schutz und zur Wiederherstellung des Umweltgleichgewichts in den Herstellungsländern finanziert werden könnten.

\subsection{TECHNOLOGIETRANSFERS}

In der Vergangenheit hatten die insgesamt recht bescheidenen Technologietransfers eine Ankurbelung des Wirtschaftswachstums in den Ländern des Südens zum Ziel, ohne dass dabei ökologischen Aspekten besondere Bedeutung beigemessen wurde. Aus zwei Gründen kommt jedoch dieser Art von Transfer bei der Verbreitung umweltfreundlicherer Produktionsmethoden in den Entwicklungsländern besondere Bedeutung zu. Erstens setzt die nachhaltige Entwicklung tiefgreifende Produktivitätsverbesserungen voraus, die nur dank leistungsfähigerer Produktionsmethoden erzielt werden können. Zweitens gewinnt der Technologiefaktor im Umgang mit der Umwelt laufend an Bedeutung. Zu erwähnen ist ebenfalls, dass die Technologietransfers aus den Industrieländern in die Entwicklungsländer nicht nur die materielle Ausrüstung, sondern auch Konstruktionsangaben, Human Resources und insbesondere die Kapazität zur Entwicklung und zum Einsatz der technischen Neuerungen umfassen. Der letztgenannte Aspekt ist für den Erfolg des Transfers von grosser Bedeutung. Der Technologietransfer beschränkt sich nicht auf die Einführung einer neuen Technologie, sondern muss ausserdem von einem Transfer des Know-How begleitet sein, das für die selbständige und fortlaufende Anpassung dieser Technologie notwendig ist.

Die Aneignung und Beherrschung ökologisch sinnvoller Technologien wurden von der UNCED als wichtige Voraussetzungen für die nachhaltige Entwicklung genannt. Daraus ergibt sich die Notwendigkeit, die Bemühungen zur Förderung und Erleichterung des Transfers solcher Technologien auf internationaler Ebene zu koordinieren. Der bisher grösste internationale Erfolg im Zusammenhang mit dem Transfer ökologisch sinnvoller Technologien wurde im Rahmen des Programms "Cleaner Production » erzielt, welches 1990 vom Programm der Vereinten Nationen für Umwelt lanciert worden war. Dieses Programm, das sich noch immer in der Entwicklungsphase befindet, leidet zur Zeit unter ernsten

${ }^{28} \mathrm{Vgl}$. H. Kox, « Integration of Environmental Externalities in International Commodity Agreements », World Development, August 1991. 
Schwierigkeiten, da weder internationale noch lokale Finanzquellen vorhanden sind. ${ }^{29}$

Abgesehen von den finanziellen Schwierigkeiten werden die Bestimmungen des Welthandes, die patentrechtlichen Regelungen sowie die Bestimmungen über Fabrikationslizenzen oft als grösstẹ Hindernisse für den Technologietransfer genannt. ${ }^{30}$ Allerdings hat sich erwiesen, dass eine Lockerung der Bestimmungen hinsichtlich des geistigen Eigentums oder der Lizenzen nicht immer zu einer quantitativen Steigerung der Technologietransfers führt. So konnte man bereits mehrmals beobachten, dass die Entwicklungsländer nicht in der Lage waren, technologisches Wissen im öffentlichen Bereich voll auszuschöpfen. Das grösste Hindernis beim Übergang zu umweltfreundlicheren Technologien in den Entwicklungsländern sind demnach nicht die Restriktionen hinsichtlich der Transfers, sondern die Kapazität zur Assimilation der Technologie. ${ }^{31}$ Der Erfolg der internationalen Kooperationsprogramme im Bereich des Transfers umweltfreundlicherer Technologien scheint je länger je mehr von der Bereitschaft der Empfängerländer abzuhängen, interne Reformen durchzusetzen, die die Anwendung und die Entwicklung dieser Technologien fördern könnten. ${ }^{32}$

Obgleich der von der öffentlichen Hand geförderte Transfer bisher nicht immer im Zeichen des Umweltschutzes stand, misst die Schweiz dem Technologietransfer innerhalb der bilateralen und der multilateralen Hilfe grosse Bedeutung bei. ${ }^{33}$ Ein weiteres Instrument in diesem Zusammenhang stellt die Exportrisikogarantie (ERG) dar. Bereits seit 1981 muss die ERG bei Exporten in die wirtschaftlich schwächsten Länder den Prinzipien der Entwicklungspolitik Rechnung tragen. Zu erwähnen ist dabei, dass $80 \%$ der Garantien auf Exporte aus der Maschinenindustrie entfallen. Der Beitrag, den dieses Instrument beim Transfer ökologisch sinnvoller Technologien leisten kann, ist daher nicht zu unterschätzen. $^{34}$

\subsection{ZUSAMMENHANG MIT DEN UMWELTPOLITIKEN DER LÄNDER DES NORDENS}

Bei Umweltproblemen auf globaler Ebene kann ein Zusammenhang zwischen der nationalen Umweltpolitik der nördlichen Industrieländer und den Bestrebungen zur Aufwertung der Umwelt in den Entwicklungsländern hergestellt werden. Ein Beispiel dafür wären die Treibhausgasemissionen. Einige westliche Länder, die sich ausserstande sehen, ihre $\mathrm{CO}_{2}$-Emissionen bis zum Jahr 2000 auf dem Niveau von $1990 \mathrm{zu}$ stabilisieren, haben eine "gemeinsame Umsetzung " («joint implementation») bei der Durchführung von Gesundungsmassnahmen

${ }^{29}$ Vgl. S. Hafez, « Financing Cleaner Production in Developing Countries », Industry and Environment, Dezember 1994, S. 75-76.

${ }^{30}$ Für eine Erörterung der Probleme im Zusammenhang mit dem geistigen Eigentum beim Technologietransfer siehe E. Mansfield, Intellectual Property Protection, Foreign Direct Investment and Technology Transfer, Washington, Weltbank, 1994.

${ }^{31}$ Vgl. C. Juma und M. Sagoff, « Policies for Technology ", in J.C. Dooge et al. (Hrsg.), An Agenda of Science for Environment and Development into the 21st Century, Cambridge, Cambridge University Press, 1992.

${ }^{32}$ Für eine detaillierte Erörterung der dafür notwendigen Reformen siehe C. Juma, « Promoting International Transfer of Environmentally Sound Technologies: The Case for National Incentive Schemes ", Green Globe Yearbook, 1994, S. 137-148.

${ }^{33}$ So wurde beispielsweise die Aufstockung eines Mischkredits für Thailand 1993 scharf kritisiert, da er die Fertigstellung eines stark umweltbelastenden Kraftwerks erlaubte. Vgl. Jahrbuch Schweiz-Dritte Welt, 1995, S. 137.

${ }^{34} \mathrm{Vgl}$. dazu L. Kébir, Mesures gouvernementales stimulant les transferts de technologie et développement durable le cas de la Suisse, Diplomarbeit DESMAP, Université de Genève, 1996. 
vorgeschlagen. Die gemeinsame Umsetzung erlaubt es einem Land, einen Teil seiner Verpflichtungen durch die Finanzierung von Massnahmen in einem Entwicklungs- oder Transitionsland zu erfüllen. Dieser Vorschlag stützt sich auf die Tatsache, dass dieselbe Reduktion schädlicher Emissionen in solchen Ländern verhältnismässig billiger ist als in den Industrieländern. Einige Pilotprojekte auf diesem Gebiet wurden bereits auf der Basis bilateraler Abkommen ins Leben gerufen (z.B. zwischen Norwegen und Mexiko). Die gemeinsame Umsetzung dürfte ein gewichtiges Argument sein, um auch die zurückhaltendsten Industrieländer zu weiteren Reduktionen der Treibhausgasemissionen zu bewegen.

In der Schweiz ist die Vorlage zur $\mathrm{CO}_{2}$-Abgabe, die 1994 vorgestellt und dem Vernehmlassungsverfahren übergeben wurde, in der Privatwirtschaft auf starken Widerstand gestossen. Diese Kreise befürchteten, dass die Einführung einer solchen Abgabe im Alleingang die Wettbewerbsfähigkeit des schweizerischen Produktionsapparates beeinträchtigen könnte. Angesichts dieser Opposition wurde die endgültige Entscheidung auf Ende dieses Jahrhunderts vertagt. Die Einführung einer solchen Abgabe käme ohnehin nur dann in Frage, wenn die Emissionen nicht über freiwillige Massnahmen reduziert werden könnten. Das neue Energiegesetz, das sich zur Zeit in der Vorbereitung befindet, soll das Ziel, die $\mathrm{CO}_{2}$-Emissionen bis zum Jahr $2000 \mathrm{zu}$ stabilisieren, in Erinnerung rufen und weitere Etappenziele für die Jahre 2005, 2010 resp. 2020 festsetzen. Ungeachtet der Instrumente, die bei der Erreichung dieser Ziele zum Einsatz kommen, dürften die Bestrebungen zur Aufwertung der Umwelt eine zunehmende Belastung für die schweizerische Wirtschaft darstellen, ist doch die Eidgenossenschaft eines der OECD-Mitglieder mit den strengsten Umweltnormen überhaupt. In diesem Zusammenhang erscheint die gemeinsame Umsetzung besonders attraktiv und verdiente es, von der Schweiz in künftigen internationalen Verhandlungen aktiv unterstützt zu werden. Sollte nach dem Jahr 2000 trotzdem eine $\mathrm{CO}_{2}$ Abgabe eingeführt werden, könnte sich ein begleitender Steuervergünstigungsmechanismus zugunsten Schweizer Unternehmen, die einen Beitrag zur Emissionsreduktion in den Entwicklungsländern leisten, als sinnvoll erweisen.

\subsection{FINANZIERUNG DER ÖKOLOGISCHEN AUFWERTUNG}

Abgesehen vom Technologietransfer wurden bisher in erster Linie jene Instrumente vorgestellt, die keiner zusätzlichen Finanzierung durch die Industrieländer bedürfen. Der heikelste Aspekt im Zusammenhang mit Entwicklung und Umwelt, nämlich derjenige der Finanzierung der ökologischen Aufwertung in den Entwicklungsländern, wurde dabei umgangen, obwohl er bei dieser Problematik eine bedeutende Rolle spielt und oft das grösste Hindernis für die Durchsetzung umweltpolitischer Massnahmen darstellt.

Als Beispiel dafür soll die Agenda 21 hinzugezogen werden (siehe oben). Das Kapitel dieses Massnahmenkatalogs über die Finanzierung der vereinbarten Programme gab Anlass zu den heftigsten Debatten. Im verabschiedeten Dokument wird hervorgehoben, dass die benötigten Ressourcen ausschliesslich über das Wirtschaftswachstum, die Öffnung des Welthandels sowie über Investitionen zusammengetragen werden können. Die Mittel sind von den Verschmutzern (mittels Abgaben und handelbarer Emissionsrechte), vom Handel sowie von der Öffentlichkeit und über freiwillige Beiträge bereitzustellen. In Anbetracht der 
Tatsache jedoch, dass bereits in den westlichen Industrieländern die wirtschaftlichen Instrumente der Umweltpolitik nur sehr langsam eingeführt werden, erscheint die Bereitstellung der vorgehend erwähnten Gelder in den Entwicklungsländern zumindest kurz- und mittelfristig bestenfalls hypothetisch. Damit in diesen Ländern Umweltprogramme rasch in die Tat umgesetzt werden können, muss vor allem auf Konzessionsfinanzierungen zurückgegriffen werden.

Die Konzessionsfinanzierung erfolgt in erster Linie über die öffentliche Entwicklungshilfe. Anlässlich des Erdgipfels in Rio haben die Industrieländer ihr Versprechen wiederholt, dabei das von den Vereinten Nationen vorgegebene Volumen von 0,7\% des BSP zu erreichen. Bisher haben die Niederlande und die skandinavischen Länder als einzige dieses Ziel erreicht. Einige Länder, darunter Deutschland und Frankreich, wollen bis zum Jahr 2000 soweit sein, andere wiederum wie etwa die USA (die die Marke von nur 0,2\% bisher nicht überschritten haben) haben sich geweigert, diese Vorgabe einzuhalten. Die Schweiz hat ihrerseits vorgesehen, bis Mitte der 90er Jahre das Budget für die Entwicklungszusammenarbeit auf $0,4 \%$ des BSP aufzustocken ${ }^{35}$, angesichts der Krise im Bundeshaushalt konnte dieses Ziel allerdings nicht umgesetzt werden. Ohne eine bedeutende Erhöhung des Budgets für die öffentliche Entwicklungshilfe dürfte es schwierig werden, Umweltprojekte durchzuführen, ohne dabei auch den Erfolg anderer nationaler Hilfsprogramme aufs Spiel zu setzen.

Gegenwärtig gibt es keinen internationalen Fonds, der die Entwicklungsländer bei der Bewältigung ihrer nationalen Umweltprobleme unterstützen könnte. Die internationale Finanzierung ökologischer Projekte von globaler Bedeutung erfolgt ihrerseits durch den GEF (Globaler Umweltfonds/Global Environment Facility), der 1990 mit einer ersten Einlage von 1,5 Milliarde Dollar in Form von Zahlungsversprechen gegründet wurde. Der GEF, der von der Weltbank, dem Entwicklungsprogramm der Vereinten Nationen (UNDP) und dem Umweltprogramm der Vereinten Nationen (UNEP) gemeinsam verwaltet wird, finanziert Projekte zur Bekämpfung der Erderwärmung, der Zerstörung der Artenvielfalt, der Verschmutzung der internationalen Gewässer und der Zerstörung der Ozonschicht. Eine Ausweitung der Tätigkeit des GEF auf den Schutz der Böden (Eindämmung der Wüstenbildung und der Abholzung) ist vorgesehen. Während der Pilotphase des GEF bestritt die Schweiz 4\% des gesamten Fonds, was einem überproportionalen Beitrag entspricht. Dies beweist, welche Bedeutung die Schweiz der Einbeziehung der Entwicklungsländer bei der Bekämpfung globaler Umweltprobleme beimisst. Die Schweiz ist aktive Teilnehmerin bei den Verhandlungen im Hinblick auf eine Umstrukturierung des GEF, die eine bessere Anpassung des Fonds an die bestehenden Abkommen zum Ziel hat.

\subsection{VERSCHULDUNG UND UMWELT}

Die UNCED hat dazu beigetragen, dass die weltweite Öffentlichkeit darauf aufmerksam wurde, dass die Finanzierung von Umweltschutzprojekten in den Drittweltländern im direkten Zusammenhang mit deren Entwicklungsrückstand und Verschuldung steht. Die Diskussion um die Erhaltung der tropischen Regenwälder ist ein ausgezeichnetes Beispiel für dieses Dilemma. In ihrer Sorge um öko-

\footnotetext{
${ }^{35}$ Vgl. Botschaft vom 15. Mai 1991 über den Beitritt der Schweiz zu den Institutionen von Bretton Woods.
} 
logische Probleme wie die Erderwärmung fordern die Industrieländer Massnahmen zur Bewahrung des Klimagleichgewichts, dessen Störung in erster Linie auf ihre eigenen übermässigen $\mathrm{CO} 2$-Emissionen zurückzuführen ist. Für die Entwicklungsländer stellt der Umweltschutz nicht immer eine absolute Priorität dar. In ihren Bemühungen, die wirtschaftliche und soziale Entwicklung voranzutreiben und die nötigen Devisen für den Schuldendienst aufzubringen, opfern sie ihre Wälder dem Bergbau, dem Tourismus, der Landwirtschaft und der holzverarbeitenden Industrie und überschreiten dabei die natürliche Regenerierungskapazität dieses wertvollen Rohstoffs. Experten schätzen, dass diese Wälder bei einer gleichbleibend intensiven Bewirtschaftung noch vor dem Jahr 2020 endgültig zerstört sein werden.

Die « Debt-for-nature-Swaps » stellen ein hervorragendes Instrument zur Finanzierung von Umweltschutzprojekten in Entwicklungländern unter gleichzeitiger Verringerung ihrer Auslandsverschuldung dar. Dabei werden Schuldbriefe, die von Umweltschutzorganen auf den Sekundärmärkten erworben worden sind, in die nationale Währung des Entwicklungslandes umgewandelt und für die Verwirklichung von Umweltschutzprogrammen im Land zur Verfügung gestellt.

Bis heute konnten lediglich etwa zwanzig solcher Abkommen mit etwa zehn Ländern geschlossen werden. Mehrere Faktoren sind für den relativen Misserfolg verantwortlich: ${ }^{36}$ Erstens bedingt das Zustandekommen eines Debt-for-nature-Swaps mehrere Akteure : eine internationale Umweltschutzorganisation (oft eine NRO), eine internationale Bank, Regierungsorgane des betreffenden Landes, die Notenbank des Landes sowie eine nationale Umweltschutzorganisation. Eine derart grosse Anzahl von Beteiligten ist dem Abschluss eines Abkommens nicht gerade förderlich. Zweitens hängt der Erfolg eines solchen Abkommens zu einem grossen Teil vom guten Willen des Empfängerlandes ab. Die Regierungen dieser Länder befürchten jedoch oft einen Verlust ihrer nationalen Souveränität zugunsten der internationalen NROs. Zudem geniessen die nationalen Umweltschutzorganisationen von Land zu Land einen anderen Status. Drittens scheuen die Notenbanken der betreffenden Länder oft davor zurück, grosse Devisensummen in Landeswährung zu konvertieren, da sie inflationäre Auswirkungen befürchten. Dieses Problem liesse sich lösen, indem diese Schulden nicht in Geld, sondern in Staatsanleihen umgewandelt werden, deren Zinsen zur Finanzierung von Umweltschutzprojekten verwendet werden könnten. Viertens müssen die Debt-for-natureSwaps, auch wenn der Diskont manchmal $85 \%$ betragen kann, dennoch auf die eine oder andere Weise finanziert werden, denn die internationalen Naturschutzorganisationen arbeiten nicht gewinnorientiert. Dies führt einmal mehr auf die bereits erörterte Frage der Finanzierung zurück. Die Finanzierung könnte durch eine einfache Entschuldungsoperation sichergestellt werden, die dem Image des Gebers, handle es sich nun um ein Land oder um eine internationale Bank, förderlich sein könnte. Seit 1991 erlässt die Schweiz den Entwicklungsländern jährlich 60-70 Millionen Franken Schulden. Die dazu benötigten Mittel stammen aus einem Kredit über 700 Millionen Franken (wovon 400 Millionen für Entschuldungsprogramme vorgesehen sind), der 1991.zum 700jährigen Bestehen der Eidgenossenschaft eröffnet wurde. Allerdings ist zu bedauern, dass diese Gelder nicht für Debt-for-Nature-Programme verwendet werden.

\footnotetext{
${ }^{36}$ Vgl. O. Schmid-Schönbein, « Debt-for-Nature Swaps : Funding Conservation in Third World Countries », Economic and Financial Prospects, Nr. 6, Dezember-Januar 1992.
} 


\section{SCHLUSSBEMERKUNGEN}

Der Umweltschutz ist ein wichtiger Bestandteil der Entwicklung. Ohne angemessene Umweltschutzmassnahmen ist keine nachhaltige Entwicklung möglich. Ohne Entwicklung gibt es keine Ressourcen für erforderliche Investitionen und folglich keinen Umweltschutz. Aber selbst wenn die Notwendigkeit der Berücksichtigung der ökologischen Komponente in der Erarbeitung von Entwicklungsprogrammen heute unbestritten ist, so reichen doch die von den Industrieländern bereitgestellten Mittel längst nicht aus, um den Ambitionen gerecht zu werden, die in Absichtserklärungen bei internationalen Konferenzen allseits geäussert werden.

Angesichts der lokalen und regionalen Umweltprobleme in den Entwicklungsländern und der Gefahr, die sie aufgrund ihrer gesundheitlichen Auswirkungen auf die Bevölkerung für die Entwicklung darstellen, sieht die Zukunft alles andere als rosig aus. Dass die Öffentlichkeit in den Entwicklungsländern diesen Problemen weniger Bedeutung beimisst als der Verschmutzung auf globaler Ebene, ist verständlich. Die Bereitstellung von Geldern für Programme zur ökologischen Aufwertung in den Entwicklungsländern gestaltet sich entsprechend schwieriger. Die Ursache dafür liegt teilweise im Wachstumsrückgang in den Industrieländern, der eine weniger « altruistische » Haltung fördert, teilweise aber auch darin, dass sich die Industriegesellschaft nicht für die Verschmutzung in den Entwicklungsländern verantwortlich fühlt. Im Hinblick auf die globalen Verschmutzungsprobleme ist die Situation weniger düster. Wie aus dem aktuellen Beispiel der Massnahmen gegen FCKW ersichtlich wird, ist die Völkergemeinschaft, angeführt von den Industrieländern, manchmal durchaus in der Lage, Massnahmen zur Verhinderung einer planetaren Katastrophe zu ergreifen, vorausgesetzt, die Gefahrenschwelle wurde klar überschritten, und die Einschätzungen der Wissenschaftler stimmen mehr oder weniger überein. Dies umso mehr, wenn der Grossteil der Verantwortung für den Missstand, wie etwa bei der Erderwärmung, klar bei den Industrieländern liegt.

Die Frage nach der Finanzierung steht im Zentrum der Problematik der ökologischen Aufwertung in der Dritten Welt. Eines ist klar : von den Entwicklungsländern selbst sind keine Wunder zu erwarten. Die Sorge um die Umwelt stellt in diesen Ländern selten ein vorrangiges Problem dar. Einige Aspekte des Umweltschutzes wie etwa der Artenschutz gelten oft als «Luxus für Reiche ». Die Finanzierung muss also von aussen kommen. Zum einen gibt es die traditionelle bilaterale und multilaterale öffentliche Entwicklungshilfe. Allerdings reicht deren Volumen nicht aus, um abgesehen von konventionellen Entwicklungsprogrammen auch noch Umweltprojekte zu finanzieren. Im besten Falle können Entwicklungshilfeprojekte einer Umweltverträglichkeitsanalyse unterzogen werden, um schädliche Auswirkungen auf ein Minimum zu begrenzen und eventuelle Synergien aufzudecken. Zum anderen existieren spezifische internationale Umweltschutzorgane und mehrere im Umweltschutz tätige NROs. Auch sie müssen auf die eine oder andere Art finanziert werden. Die Umwandlung von Schulden der Drittweltländer in Gelder, die für Umweltschutzmassnahmen bereitgestellt werden, stellt eine Lösung dar, die bisher nicht voll ausgeschöpft wurde. Eine weitere, umstrittene Möglichkeit wäre die Finanzierung dieser Organe über eine international erhobene Abgabe auf verschmutzende Rohstoffe oder auf Geldgeschäfte. Und schliesslich gibt es die leider noch kaum umgesetzte Mög- 
lichkeit, Private vermehrt zu Beitragszahlungen zu bewegen. Dazu wäre natürlich ein Anreizmechanismus nötig. Dieser Anreiz könnte steuerlicher Natur sein, indem man Unternehmen aus den Industrieländern die Möglichkeit bietet, Investitionsausgaben für Umweltschutzmassnahmen, die in den Entwicklungsländern getätigt wurden, von den Steuern abzusetzen. Ausserdem könnte man Patenschaftsverträge mit Unternehmen aushandeln, die dank ihrer finanziellen Unterstützung zugunsten eines Umweltschutzprojektes wie z.B. eines Nationalparks in einem Entwicklungsland Imagewerbung betreiben könnten. 